\title{
A 4-week comparison of capillaroscopy changes, healing effect, and cost-effectiveness of botulinum toxin-A vs prostaglandin analog infusion in refractory digital ulcers in systemic sclerosis
}

\author{
Saeedeh Shenavandeh ${ }^{1}$ D $\cdot$ Mozhdeh Sepaskhah $^{2} \cdot$ Sanaz Dehghani $^{3} \cdot$ MohammadAli Nazarinia $^{1}$
}

Received: 23 April 2021 / Revised: 25 August 2021 / Accepted: 28 August 2021 / Published online: 1 September 2021

(c) International League of Associations for Rheumatology (ILAR) 2021

\begin{abstract}
Introduction Systemic sclerosis (SSc) is a systemic multi-organ disease. Raynaud's phenomenon (RP) and digital ulcers (DUs) in SSc patients can be resistant to usual treatments. We studied the clinical benefits, capillaroscopy changes, and costeffectiveness of local injection of botulinum toxin-A (BTX-A) and intravenous prostaglandin analogs (iloprost/alprostadil) in patients with SSc with resistant DUs.

Method In a clinical trial study, we evaluated 26 patients fulfilling the ACR/EULAR SSc criteria with resistant DUs. Visual analog scale of pain and RP, skin color and type of ulcers, and capillaroscopy were assessed before and 1 month after treatment. In the first group, 20 units of BTX-A was injected at the base of each involved fingers by a dermatologist. In the second group, $20 \mu \mathrm{g}$ iloprost or $60 \mu \mathrm{g}$ alprostadil was infused daily. The cost of these treatments was compared.

Result In 26 patients (43 fingers), there were 16 patients ( 22 fingers) in the BTX-A and 10 patients ( 21 fingers) in the prostaglandin group. In $95.5 \%$ of the BTX-A and $90.5 \%$ of the prostaglandin group, the ulcers were healed. In both groups, a significant decrease in pain was seen $(p<0.0001)$. Capillaroscopy patterns in both groups were not changed although the microhemorrhages disappeared significantly ( $p$ value: BTX-A: 0.03 and prostaglandin: 0.002 ). The cost was significantly lower in the BTX-A injection group $(p<0.0001)$.

Conclusion Both BTX-A and prostaglandins helped in the healing and pain control of DUs. In capillaroscopy, microhemorrhages were significantly decreased in both groups. In the BTX-A group, the cost was significantly lower as an outpatient treatment and was more time-saving.
\end{abstract}

\section{Key Points}

- BTX-A and prostaglandin analogs both contributed to the healing

of digital tip ulcers and improving the pain

- In capillaroscopy, microhemorrhages were significantly decreased

or disappeared after both treatments

- There was no significant side effect in both groups

- Comparing both groups, in the BTX-A group, the cost was sig-

nificantly lower when performed on an outpatient treatment and

more time-saving.

Keywords Botulinum toxin $\cdot$ Capillaroscopy $\cdot$ Digital ulcer $\cdot$ Prostaglandin analogs $\cdot$ Systemic sclerosis

Saeedeh Shenavandeh

shenavande@sums.ac.ir

1 Department of Internal Medicine, Division of Rheumatology, Namazee Hospital, Shiraz University of Medical Sciences, 71345-1414 Shiraz, Iran

2 Molecular Dermatology Research Center, Shiraz University of Medical Sciences, Shiraz, Iran

3 Department of Internal Medicine, Shiraz University of Medical Sciences, Shiraz, Iran

\section{Introduction}

Systemic sclerosis (SSc) is a connective tissue disease characterized by deposition of collagen and other components of extracellular matrix in the skin and internal organs, microvascular damage, and fibrosis of the skin and internal organs along with activation of the immune system [1], leading to local complications like digital ulcers 
and pitting scars that are sometimes very troublesome and resistant to treatment. Digital ulcers (DUs) have been reported in nearly $50 \%$ of patients with SSc with a high frequency of recurrence and resistance to treatments [2]. It was shown that almost $75 \%$ of patients had their first DUs within 5 years from their first non-Raynaud symptom [3], sometimes leading to tissue loss, osteomyelitis, gangrene, septicemia, auto-amputation, impaired hand function, and decreased quality of life [3, 4]. Multiple pharmacological therapies for the treatment and prevention of both Raynaud's phenomenon (RP) and DUs including calcium channel blockers, antiplatelet agents, endothelin receptor antagonists, phosphodiesterase inhibitors, and statins have been suggested [5].

In patients with SSc, iloprost (prostaglandin I2 analog) and alprostadil (prostaglandin E1 analog) have been reported to be effective on RP, skin score, and DUs [6]. Specifically, in more than half of the cases treated with iloprost, a decrease in the time of healing and a reduction in formation of new DUs were displayed [7]. The mechanism of their clinical advantages was described as vasodilatation, antiplatelet activity that persists for just a few hours after infusion, enhancement of fibrinolysis, interference with immune response, modulation of the cytokine network, and influence on endothelial functions with longer effect [8]. There is preliminary evidence that local injection of botulinum toxin (BTX-A), which is a neurotoxin generated by the Gram-positive anaerobic bacteria, Clostridium botulinum, and probably inhibits sympathetic nerve activity [9], has beneficial effects in the treatment of RP and digital ischemic complications. Improved vascularity (tissue perfusion) and relief of pain and ulcerations have been reported by many patients in all of researches and many patients stayed painless for many months [10]. However, its use is mostly based on open-label, uncontrolled studies, and more rigorous clinical analyses have been recommended.

Peripheral microangiopathy can be easily recognized by nailfold capillaroscopy, a noninvasive and safe method that is well established in investigation of patients with RP and SSc [11]. It has been shown that there are associations between some evolutional microvascular changes seen in capillaroscopy of patients with SSc during followup, like progressive reduction of the number of capillaries and some disease manifestations including development of new DUs, pulmonary vascular hypertension, and skin fibrosis(12).

In this study, we made an attempt to determine the healing effect and nailfold capillary changes before and after BTX-A injection in resistant digital ischemic ulcers of patient with SSc as a cheaper and outpatient technique compared to inpatient intravenous prostaglandin analog treatments that are recommended in patients with resistant digital ulcers.

\section{Materials and methods}

In this study, we evaluated the patients with SSc who fulfilled 2013 ACR/EULAR SSc diagnostic criteria [13] for SSc with resistant active ischemic digital ulcers in their hands, referring to Hafez Hospital Clinic of scleroderma and the patients who have been admitted in Hafez Rheumatology Ward affiliated to Shiraz University of Medical Sciences, from December 2017 to December 2019. Sampling method was census method. Then, we divided the patients into two groups after fulfillment of the inclusion criteria and lack of the exclusion criteria. The first group consisted of patients with SSc and digital ulcers in their hands that referred to a dermatologist for local botulinum toxin-A (BTX-A) injection, and the second group comprised the patients with SSc and digital ulcers in their hands who were admitted to receive alprostadil or iloprost intravenous infusion and followed for 1 month after that. Clinical manifestations of patients and their organ involvement were recorded during evaluation.

\section{Inclusion criteria}

The study groups consisted of SSc patients aged more than 16 years old and less than 60 years old. The patient selection was based on ACR-EULAR criteria [13] for SSc. The patients with at least one active distal ischemic digital ulcer (DU) (defined as those with a visible de-epithelialized base; although, if denudation was not clearly visible, loss of epithelialization was allowed to be "determined" by the investigator) on the volar or lateral sides of the distal fingers were included in the study. Only digital ulcers distal to the proximal interphalangeal joints on the volar and lateral sides were assessed. Other treatments like calcium channel blockers, antiplatelet agents, PDE-5 inhibitors, and endothelin receptor antagonists were not discontinued. Prostaglandin analogs should had been received more than 2 months before the study time and were not used during the 1-month study time in the group that underwent BTX-A injections. The patients should had used at least two highest tolerable doses of a dihydropyridine calcium channel blocker and/or PDE-5 inhibitors before this trial study without response.

\section{Exclusion criteria}

Cases with overlap syndrome; who are smoker, pregnant, and lactating; and with diabetes mellitus were excluded from the study. Patients with vascular insufficiency leading to ischemia of digits shown in color Doppler sonography of the affected arm or with abnormal peripheral pulses were excluded from this study. Patients with infective ulcers or 
calcinosis-induced ulcers and patients with history of previous sympathectomy were excluded too. Patients with ulcers due to fissures, ulcers over the calcinosis area, not painful ulcers, and undetermined and gangrenous ulcers were also excluded. Gangrenous lesions at the fingertip in which the tip of the digit was dry, shrunken, and dark black were not considered ulcers, so they were excluded. Patients without available nailbeds (at least in fingers with digital ulcers) for capillaroscopy were also excluded. All the patients were required to sign a declaration of consent, before being included in the study population.

\section{Method of study}

Informed consent was obtained from all participants. The patients who were referred to scleroderma outpatient clinic and fulfilled the inclusion criteria, refused to be admitted in hospital, and agreed for BTX-A injection were referred to a dermatology clinic. The patients with resistant digital ulcers who were admitted by their physicians in hospital for receiving infusion of prostaglandin analogs and agreed to do the capillaroscopy and be followed after 1 month were included too. Physical examination was done by a rheumatologist involved in this research and the data were saved in a chart. The SSc patients were assessed for sex, age, disease duration, and clinical (diffuse or limited) subsets according to (2013) ACR/EULAR SSc diagnostic criteria [13]. The digit color was described relative to the adjacent uninvolved digits. The involved fingers were assessed under ambient room light when placed on a white background as normal or cyanotic. Pain and number of attacks of RP in the last 2 weeks were assessed by visual analog scale (VAS). Skin color and type of ulcers (no ulcer, active ulcer, indeterminate ulcer (definite ulcers with a base that was not visible), and healed ulcer (when total re-epithelialization of an ulcer was observed)) were also registered before and 1 month after therapy.

Outcome parameters and at least one picture from the involved fingers were assessed at baseline (pretreatment) and 1 month post-injection of BTX-A or infusion of prostaglandin analogs.

On the same day, both groups underwent capillaroscopy, measuring the VAS of pain and a picture of their ulcerated fingers, and after 1 month the patients had another visit for having another picture of the digit and VAS of pain measurement and capillaroscopy of the ulcerated fingers. Nailfold capillaroscopy and the measurements were done by the rheumatologist involved in the study using the stereomicroscope Euromex ST. 1740 made in Holland by X250 power and video camera Cmex D.C.5000 (5 megapixels). The data were recorded in a form including distribution (normal or disturbed (mild, moderate, severe)); shape of capillaries (normal or abnormal shape including ramification, neoangiogenesis, and branching); capillary diameter: the largest diameter of apical side of the nailfold capillaries (if it was $\geq 20 \mu \mathrm{m}$, it was marked as capillary dilatation and if it was $\geq 50 \mu \mathrm{m}$, it was marked as giant loop); capillary length (mean of the 3 highest length $\geq 300 \mu \mathrm{m}$ was called elongated); mean capillary density (the mean capillary density in $1 \mathrm{~mm}>9$ capillaries/mm = very good density, between 7 and 9 capillaries $/ \mathrm{mm}=$ good density, between 4 and 6 capillaries $/ \mathrm{mm}=$ reduced (low) density, $<4$ capillaries $/ \mathrm{mm}=$ very low density); and presence or absence of avascular areas and hemorrhage in the involved fingers based on the international Delphi consensus for reporting the data and the PANLAR capillaroscopy study group consensus [14].

The overall capillaroscopy patterns were defined as normal, non-specific abnormalities [15], and scleroderma patterns (including early, active, and late pattern) using Cutolo et al.'s capillaroscopy classifications and standardization of nailfold capillaroscopy $2020[16,17]$.

The pictures of all affected nailfolds' capillaroscopy and all affected ulcerated fingers in each visit (first and after 1 month) were saved.

The criteria of improvement on capillaroscopy were as follows: reduction in hypooxygenation which was recognized by decreased branching (abnormal shapes) or changing from late pattern of capillaroscopy to active or early pattern; increased number of capillary loops per millimeter; decreased number of giant loops and microhemorrhages; and regression from active form to early pattern of capillaroscopy and changing from fully disturbed distribution to normal or mild disturbed distribution.

The patients in the first group were sent to Imam Reza Clinic related to Shiraz University of Medical Sciences to have BTX-A injection by the dermatologist involved in this study. The dermatologist diluted 500 units of botulinum toxin-A (abobotulinumtoxin-A (Dysport $\left.{ }^{\circledR}\right)$, Ipsen, UK) in $2.5 \mathrm{~mL}$ of normal saline, and after local anesthesia of the sites of injection by application of lidocaine-prilocaine cream (Xyla P®) (Tehran Chemie, Tehran, Iran) for $1 \mathrm{~h}$ under plastic wrap (under occlusion to enhance anesthetic effect), she injected both the medial and lateral sides of the root of every involved digit (adjacent to the neurovascular bundles, at the root of bilateral palmar proper arteries of each involved finger) with 31 gauge, $6 \mathrm{~mm}$, Micro-Fine ${ }^{\mathrm{TM}}$ plus insulin syringe (Becton Dickinson (BD), USA) with aseptic technique. Injection dosage was 10 units at each point (20 units for each finger). The second group of the patients with digital ulcer were admitted in the rheumatology ward of Hafez Hospital and received either iloprost (Ilomedin, Bayer Schering Pharma, $20 \mathrm{mcg} / \mathrm{mL}$ ) as daily infusion of $20 \mu \mathrm{g}$ during $24 \mathrm{~h}$ for 3 days or alprostadil $(60 \mu \mathrm{g}$ daily during $10 \mathrm{~h}$ for 3 days (Genfarma, $20 \mathrm{mcg}$ injection, powder, lyophilized) or continuous $250 \mu \mathrm{g}$ intravenous infusion (Pfizer, $500 \mathrm{mcg} / \mathrm{mL}$ ) during $48 \mathrm{~h}$ ). 


\section{Statistical analysis methods}

Assessment and analysis were done using SPSS-16 for windows and variation analysis. Statistical analysis was performed by the use of mean, standard deviation, percentage, and the Fisher exact test. The confidence interval of $95 \%$ was considered, and $p<0.05$ was the statistical significance level adopted. Descriptive statistics method involves the use of descriptive statistics like mean, standard deviation, frequency, and frequency percent. For inferential statistics, two independent sample $t$-test and paired-sample $t$-test were used; otherwise, if data did not have normal distribution, we used the Mann-Whitney and Wilcoxon.

\section{Results}

In this study, among the 29 cases that were included and received their individualized treatments, due to no follow-up after 1 month, three patients were excluded (although they reported improvement of their ulcers and mostly returned for their routine visit after 2 or 3 months). So 16 patients (22 fingers with digital ulcers) in the first group and 10 patients (21 fingers with digital ulcers) in the second group completed the trial and enrolled in our study. Figure 1 shows the number of patients, excluded ones, and patients' categorization during the study period.

The demographic data, clinical manifestations, and the medications of study groups are shown in Table 1 .
We analyzed the clinical manifestations and capillaroscopic patterns in both groups (Table 2; Figs. 2 and 3, and supplementary images).

In the first group before the treatment with BTX-A, all of the patients had digital tip ulcer and after that the ulcer disappeared in $95.5 \%$ of them ( $p$ value $<0.0001$ ); also, in the second group, all patients had digital tip ulcer before using prostaglandin analogs and after that the ulcers disappeared in $90.5 \%$ of them ( $p$ value $<0.0001$ ).

In the first group, digital pain decreased from 4.9 over 10 to 2.54 over 10 after injecting BTX-A ( $p$ value $<0.0001$ ), and in the second group digital pain decreased from 5.05 over 10 to 2.67 over 10 after treatment with prostaglandin analogs ( $p$ value $<0.0001$ ). Both treatments also led to disappearance of cyanosis of the digits, too $(p$ value $<0.0001)$.

Injecting BTX-A and prostaglandin analogs had no significant effect on digital ischemia and RP ( $p$ value $>0.05$ ). Capillaroscopy pattern in both groups was mostly scleroderma pattern with dominant active pattern. The scleroderma patterns including early, active, and late pattern in both groups did not show significant changes ( $p$ value $>0.05$ ).

In evaluating the capillary components of capillaroscopy, hemorrhage disappeared significantly in both groups ( $p$ value $=0.03$ and 0.002 , respectively). There were no significant changes in capillary distribution, morphology, dimension, density, presence of elongation, avascular area, and abnormal shapes before and after receiving treatment in both groups ( $p$ value $>0.05$ ).
Fig. 1 Diagram of patients with digital ulcers in systemic sclerosis, total number at the study beginning, and changes during study period

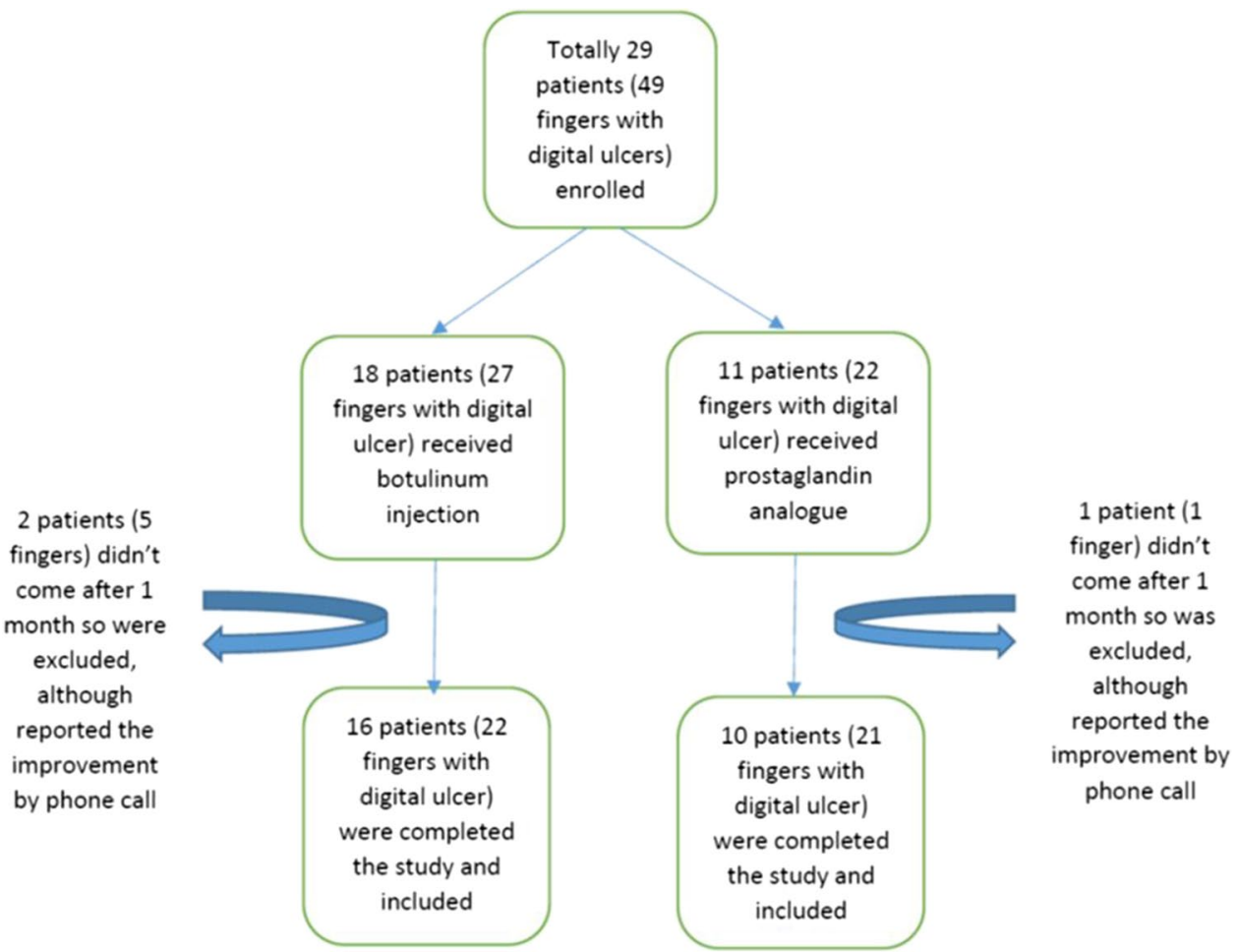


Table 1 Demographic, clinical manifestations, and medications of study groups

Group 1 (botulinum), N: 16 (22 fingers)
Group 2 (prostaglandin analogs), $N: 10$ (21 fingers)

\begin{tabular}{|c|c|c|}
\hline \multicolumn{3}{|l|}{ Demographic characteristics } \\
\hline Women, $n(\%)$ & $11(68.7)$ & $9(90)$ \\
\hline Men, $n(\%)$ & $5(31.2)$ & $1(10)$ \\
\hline Age, median (range) & $39.5(25-60)$ & $51.5(28-63)$ \\
\hline Duration of disease from Raynaud's (years), median (range) & $8(1-25)$ & $11(3-35)$ \\
\hline \multicolumn{3}{|l|}{ Clinical features } \\
\hline Diffuse SSc, $n(\%)$ & $5(31.2)$ & $1(10)$ \\
\hline Limited SSc, $n(\%)$ & $11(68.7)$ & $9(90)$ \\
\hline Raynaud's, $n(\%)$ & $16(100)$ & $10(100)$ \\
\hline Digital ulcers, $n(\%)$ & $16(100)$ & $10(100)$ \\
\hline Cardiac involvement, $n(\%)$ & $0(0)$ & $0(0)$ \\
\hline Gastrointestinal involvement, $n(\%)$ & $14(87.5)$ & $9(90)$ \\
\hline Interstitial lung disease, $n(\%)$ & $11(68.7)$ & $6(60)$ \\
\hline Pulmonary hypertension (PAP > $35 \mathrm{mmHg}), n(\%)$ & $0(0)$ & $0(0)$ \\
\hline Modified Rodnan skin score, median (range) & $14(4-28)$ & $14.5(4-23)$ \\
\hline Finger to palm limitation, $n(\%)$ & $5(31.2)$ & $5(50)$ \\
\hline \multicolumn{3}{|l|}{ Medications } \\
\hline Prednisolone, $n(\%)$ & $14(87.5)$ & $5(50)$ \\
\hline Rituximab, $n(\%)$ & $4(25)$ & $1(10)$ \\
\hline Aspirin, $n(\%)$ & $14(87.5)$ & $5(50)$ \\
\hline Methotrexate, $n(\%)$ & $2(12.5)$ & $1(10)$ \\
\hline Mycophenolate mofetil, $n(\%)$ & $4(25)$ & $4(40)$ \\
\hline Pentoxifylline, $n(\%)$ & $5(31.2)$ & $2(20)$ \\
\hline Calcium channel blockers, $n(\%)$ & $16(100)$ & $10(100)$ \\
\hline Sildenafil or tadalafil, $n(\%)$ & $7(43.7)$ & $3(30)$ \\
\hline
\end{tabular}

Data are $n(\%)$ unless otherwise indicated. SSc, systemic sclerosis; PAP, pulmonary artery hypertension

In evaluating the side effects and complications of BTX-A treatment, we encountered post-injection weakness for about 1-2 $\mathrm{h}$ in (2 fingers, 2 patients) $9 \%$ of fingers and $12.5 \%$ of patients and post-injection numbness for about $1-2 \mathrm{~h}$ in (1 finger, 1 patient) $4.5 \%$ of the fingers and $6.2 \%$ of the participants in the first group due the effect of topical anesthetic cream in these patients. In one patient ( $4.5 \%$ of fingers) after injecting BTX-A, a local finger infection on a previous knuckle ulcer rather than on the site of injection or previous distal digital tip ulcer was seen, which was healed with oral and topical antibiotic therapy. In the second group, we did not observe any significant complication.

The average cost in the first group that was treated with botulinum toxin was 76,700 Tomans (between 42,000 and 164,700 Tomans (mean $=103,350$ Tomans relatively equal to 4 dollars) for each patient. The cost of botulinum toxin injection for each finger was 44,000 Tomans and the mean number of fingers in each patient was about 1.6 fingers.

The average cost in the control group that was treated with prostaglandin analogs was 1,195,000 Tomans (range:
$1,072,000$ to 3,510,000 Tomans ( $m e a n=2,291,000$ Tomans equal to 92 dollars), including the admission cost staying about 3-5 nights in hospital for every patient). The cost was significantly more in the second group that was treated with prostaglandin analogs $(p$ value $<0.0001)$.

\section{Discussion}

In this study, we evaluated the clinical improvement, capillaroscopy changes, and cost of one time botulinum toxin injection in the outpatient clinic and intravenous prostaglandin analog infusion during hospital admission of resistant digital tip ulcers in patients with SSc who did not respond to previous medications. In both groups, we noticed significant improvement of digital ulcers and pain and a significant decrease in microhemorrhages in capillaroscopy, and a significant cost difference.

In a review study on 5 previous articles that evaluated the effect of botulinum toxin injection on patients with SSc 


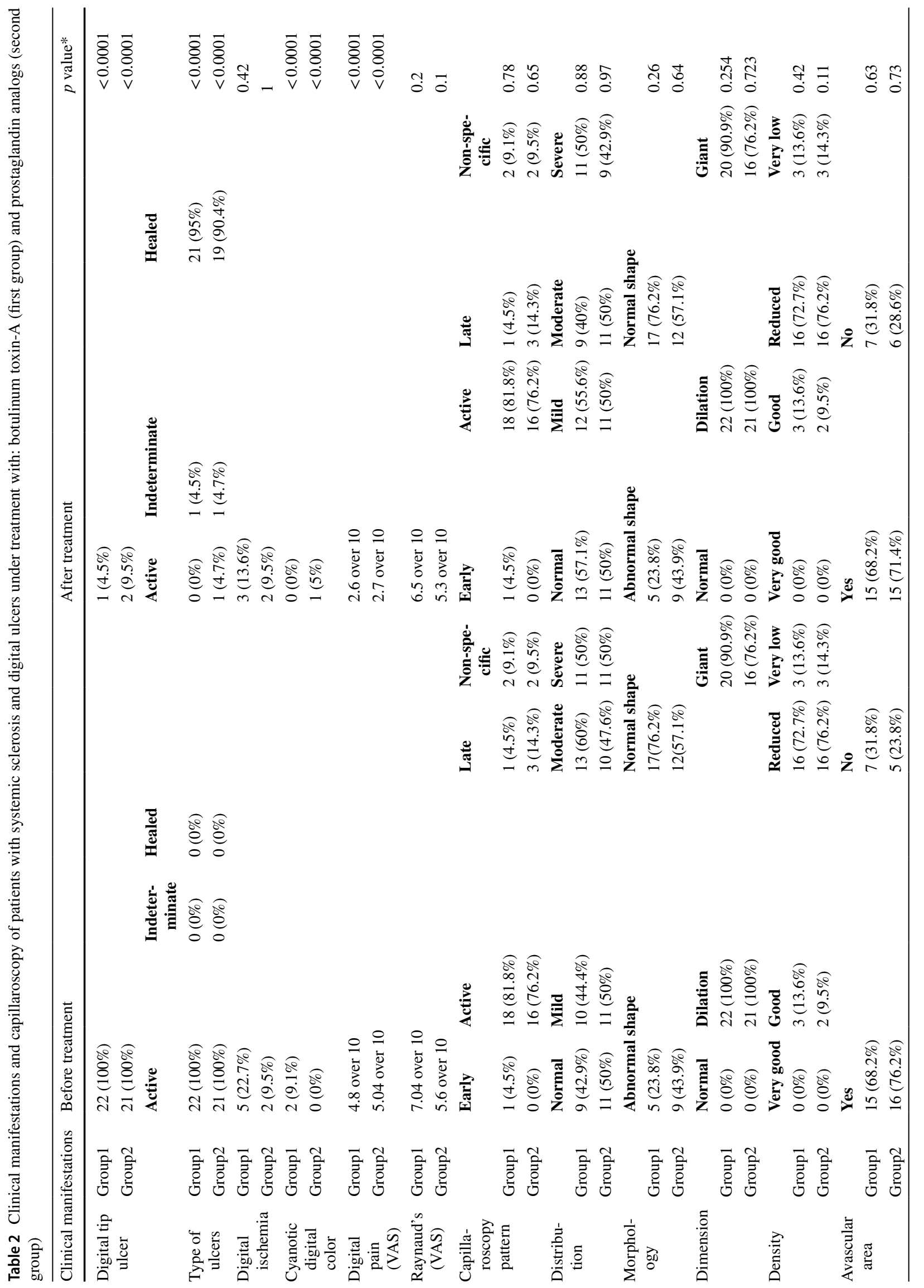



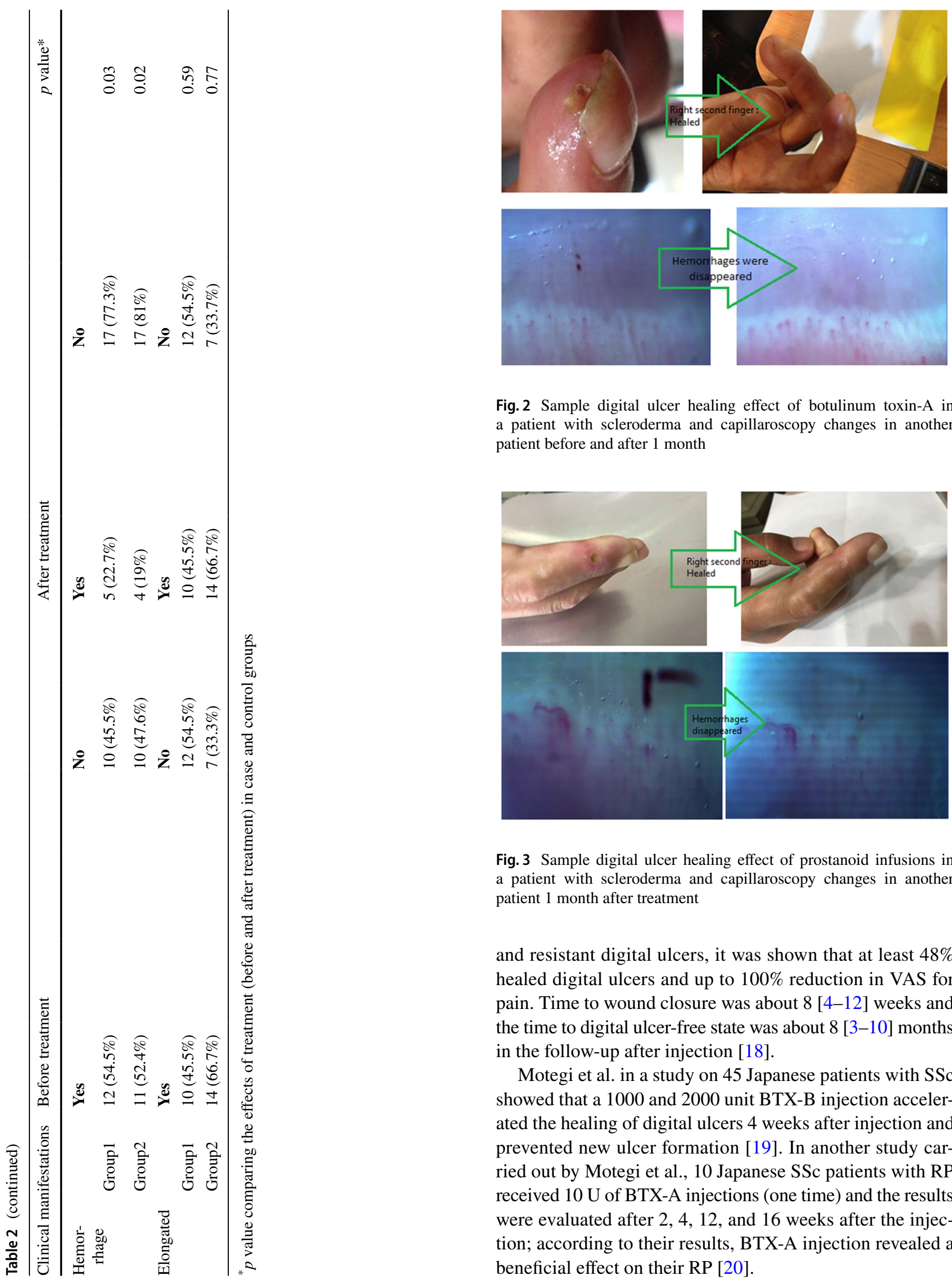

Fig. 2 Sample digital ulcer healing effect of botulinum toxin-A in a patient with scleroderma and capillaroscopy changes in another patient before and after 1 month

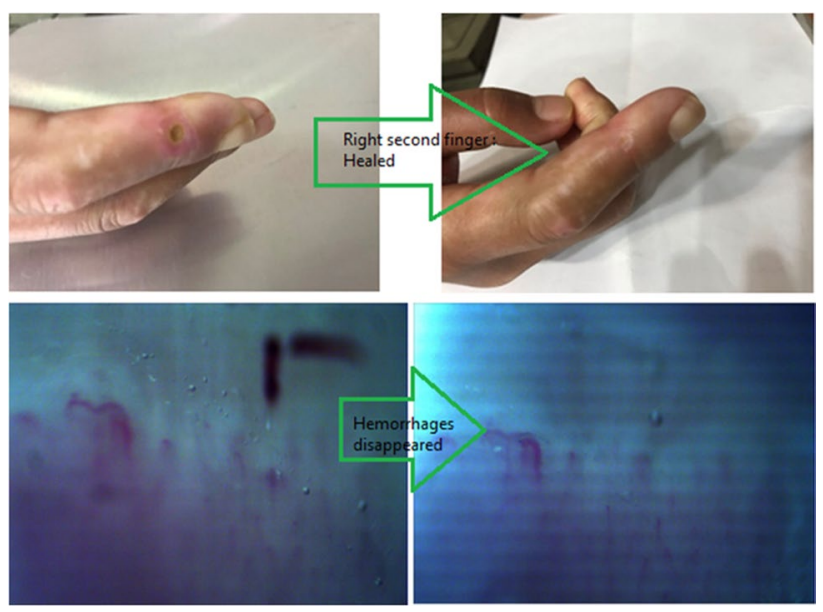

Fig. 3 Sample digital ulcer healing effect of prostanoid infusions in a patient with scleroderma and capillaroscopy changes in another patient 1 month after treatment

and resistant digital ulcers, it was shown that at least $48 \%$ healed digital ulcers and up to $100 \%$ reduction in VAS for pain. Time to wound closure was about 8 [4-12] weeks and the time to digital ulcer-free state was about 8 [3-10] months in the follow-up after injection [18].

Motegi et al. in a study on 45 Japanese patients with SSc showed that a 1000 and 2000 unit BTX-B injection accelerated the healing of digital ulcers 4 weeks after injection and prevented new ulcer formation [19]. In another study carried out by Motegi et al., 10 Japanese SSc patients with RP received $10 \mathrm{U}$ of BTX-A injections (one time) and the results were evaluated after $2,4,12$, and 16 weeks after the injection; according to their results, BTX-A injection revealed a beneficial effect on their RP [20]. 
Iloprost as a prostacyclin analog given intravenously was evaluated in a meta-analysis conducted by Tingey in 2013, and they showed that IV iloprost had a statistically significant effect in preventing new digital ulcer formation in patients with $\mathrm{SSc}(21)$. In one study done by Wigley et al. on 131 patients with $\mathrm{SSc}$, the patients received 5-day (6 h per day) intravenous infusions of iloprost ( 0.5 to $2.0 \mathrm{ng} / \mathrm{kg}$ per min); follow-up visits were scheduled at 3, 6, and 9 weeks after completion of the infusion and it was shown that iloprost could heal digital lesions [22].

In our study, in both groups after treatment, digital pain of the ulcers also decreased significantly. In a study conducted by Neumeister et al., 33 patients with RP were injected with 50 to $100 \mathrm{U}$ of BTX-A. All patients experienced relief of pain [23]. In 2004, Sycha et al. also reported improvement of pain, numbness, and cutaneous blood flow by laser Doppler imaging after intra-digital Botox injections [10].

Contrary to the results of the present study which showed that both treatments had no significant effect on RP and digital ischemia after 1 month of follow-up, numerous studies have shown that botulinum toxin injection has a positive effect on RP. It was suggested that BTX-A-induced acetylcholine-mediated vascular smooth muscle paralysis may inhibit spasm and vascular contraction and increase the blood flow. This mechanism is thought to contribute to the improvement of RP by BTX-A (2 weeks after injection of $10 \mathrm{U}$ of BTX-A) [24]. In a study by Fregene et al., 10 to 100 botulinum toxin was injected in 31 patients with digital ischemia and the patients were observed for an average of 18 months; they reported that botulinum toxin injection was safe and useful for vasospastic digital ischemia in patients with RP [10]. In another study that was done on 29 patients with RP by McHugh et al., 6-h infusions of iloprost or placebo on 3 successive days with 6 weeks between treatment periods were studied and they showed that there was no difference between iloprost and placebo in healing of ischemic lesions in patients with RP, but the frequency and severity of RP attacks were decreased with iloprost infusion [6]. In 1994, Wigley et al. also studied 131 patients with SSc. Patients received 6-h intravenous infusions of iloprost $(0.5$ to $2 \mathrm{ng} / \mathrm{kg}$ per min) or a similar volume of placebo. The patients were followed for 9 weeks. They showed no differences between iloprost and placebo in the frequency or severity of RP [22]. We think that differences in the number of infusions and the duration of follow-up may also cause these differences in the results.

In our study, the capillaroscopy pattern in both groups was mostly scleroderma pattern with dominant active scleroderma pattern that was not changed significantly in both groups after treatment; although, hemorrhages decreased or disappeared in both groups. In a study by Schioppo et al., no effect of 1 day or consecutive 5 days of iloprost infusions was shown on capillary numbers $/ \mathrm{mm}$ in capillaroscopy after 1 month of treatment [25]. Also, in another study by Cestelli et al., after 1 year of follow-up of patients who received iloprost monotherapy infusion, they showed a significant reduction of microhemorrhages [26]. In another study on the effects of treatment with bosentan and iloprost on nailfold absolute capillary number compared to only 5 days of iloprost infusions after 4 years, the patients showed a progressive absolute capillary number/ $\mathrm{mm}$ decrease in the iloprost monotherapy; however, in bosentan and iloprost combination therapy, they showed an increase in capillary numbers, so it seemed to be effective only when used in combination [27]. A case report study also showed improvement of dermatoscopy features of two patients with SSc after 12-16 weeks of injection of Botox ${ }^{\circledR}$ with decreased capillary loss or increased dilated loops [28].

The average cost in the first group treated with BTX-A was significantly lower than the cost of prostaglandin ana$\log$ s, including the admission cost of staying about 3-5 nights in hospital for every patients. So outpatient treatment with botulinum toxin was effective and can be a good choice and more cost-effective.

In evaluating the side effects and complications of BTX-A treatment, we saw post-injection temporary weakness for about $1-2 \mathrm{~h}$ and post-injection numbness for about $1-2 \mathrm{~h}$ in two and one patients respectively and a localized ulcer infection not at the site of injection of botulinum toxin in one patient, which was treated with oral and topical antibiotic therapy.

Therefore, based on our study and the significant therapeutic effects of both BTX-A and prostaglandin analog infusions in clinical relief of digital ulcers and pain and also decrease in microhemorrhages in capillaroscopy, it seems both treatments are helpful in acute management of resistant DUs of patients with SSc although using BTX-A local injections is more cost-effective and time-saving for patients with SSc as it needs once clinic referral for injection, especially in this era of the COVID-19 pandemic that patients prefer to have shorter and lower referrals and lower admission rates. In our experience, following the patients with BTX-A injection, after their good experience, during their future recurrence of digital ulcers in cold weather, they mostly asked for BTX-A injections for their pain relief and digital ulcer healing, while denying further admissions, especially in this era of COVID-19 too that we will discuss it in further studies. The limitations of our study were limited follow-up time (only 1 month) and use of only one time injection for each patient. Probably, in follow-up studies, the evaluation of more injection sessions of BTX-A or combining it with prostaglandin analogs and having more follow-up months will help the patients in the prevention of recurrence of their ulcers. 


\section{Conclusion}

Our study showed both BTX-A and prostaglandin analogs contributed to healing of digital tip ulcers and improving the pain of patients with SSc coming with resistant digital ulcers. In capillaroscopy, microhemorrhages were significantly decreased or disappeared after treatment. There was no significant side effect in both groups. In the BTX-A group, the cost was significantly lower when performed on an outpatient treatment and was more time-saving.

Supplementary Information The online version contains supplementary material available at https://doi.org/10.1007/s10067-021-05900-7.

Acknowledgements The authors would like to thank Shiraz University of Medical Sciences, Shiraz, Iran, and also Center for Development of Clinical Research of Namazee Hospital and Dr. Nasrin Shokrpour for editorial assistance.

Funding This thesis was supported by the dean of School of Medicine based on research project number 1396-01-01-15726 and sponsored by the vice chancellor of Shiraz University of Medical Sciences.

Data availability All data and materials of this manuscript are available from the corresponding author on reasonable request.

\section{Compliance with ethical standards}

Ethics approval and consent to participate The study was approved by the Human Ethics Review Committee of our University of Medical Science number: IR.SUMS.MED.REC.1398.360. Informed consent was obtained from all the participants before entering the study. There was no animal study in this article.

Consent for publication Not applicable.

Disclosures None.

\section{References}

1. Fredrick M. Wigley MD aNAF, Ph.D. 2016 Raynaud's phenomenon. The New England Journal of Medicine

2. Polito P, Zanatta E, Felicetti M, Benvenuti F, Favaro M, Cozzi F et al (2020) Skin ulcers in systemic sclerosis: correlation with clinical phenotype in a monocentric cohort from the north-east of Italy. Clin Exp Rheumatol. 38(Suppl 125 (3)):148-53

3. Nihtyanova SI, Brough GM, Black CM, Denton CP (2008) Clinical burden of digital vasculopathy in limited and diffuse cutaneous systemic sclerosis. Ann Rheum Dis 67(1):120-123

4. Hachulla E, Clerson P, Launay D, Lambert M, Morell-Dubois S, Queyrel V et al (2007) Natural history of ischemic digital ulcers in systemic sclerosis: single-center retrospective longitudinal study. J Rheumatol 34(12):2423-2430

5. Chung L, Fiorentino D (2006) A pilot trial of treprostinil for the treatment and prevention of digital ulcers in patients with systemic sclerosis. J Am Acad Dermatol 54(5):880-882

6. McHugh NJ, Csuka M, Watson H, Belcher G, Amadi A, Ring EF et al (1988) Infusion of iloprost, a prostacyclin analogue, for treatment of Raynaud's phenomenon in systemic sclerosis. Ann Rheum Dis 47(1):43-47

7. Ho M, Veale D, Eastmond C, Nuki G, Belch J (2000) Macrovascular disease and systemic sclerosis. Ann Rheum Dis 59(1):39-43

8. Gardinali M, Pozzi MR, Bernareggi M, Montani N, Allevi E, Catena $L$ et al (2001) Treatment of Raynaud's phenomenon with intravenous prostaglandin E1alpha-cyclodextrin improves endothelial cell injury in systemic sclerosis. J Rheumatol 28(4):786-794

9. Aoki KR (2001) Pharmacology and immunology of botulinum toxin serotypes. J Neurol 248(Suppl 1):3-10

10. Fregene A, Ditmars D, Siddiqui A (2009) Botulinum toxin type A: a treatment option for digital ischemia in patients with Raynaud's phenomenon. J Hand Surg Am 34(3):446-452

11. Sato LT, Kayser C, Andrade LE (2009) Nailfold capillaroscopy abnormalities correlate with cutaneous and visceral involvement in systemic sclerosis patients. Acta Reumatol Port 34(2A):219-227

12. Avouac J, L G, Smith V, Toniolo E (2017) Sequential nailfold videocapillaroscopy examinations have responsiveness to detect organ progression in systemic sclerosis. Seminars in Arthritis and Rheumatism. 47(1):86-94

13. van den Hoogen F, Khanna D, Fransen J, Johnson SR, Baron M, Tyndall A et al (2013) 2013 classification criteria for systemic sclerosis: an American College of Rheumatology/European League against Rheumatism collaborative initiative. Arthritis Rheum 65(11):2737-2747

14. Ingegnoli F, Herrick AL, Schioppo T, Bartoli F, Ughi N, Pauling JD, et al. 2020 Reporting items for capillaroscopy in clinical research on musculoskeletal diseases: a systematic review and international Delphi consensus. Rheumatology (Oxford)

15. Corrado A CG, Gaudio A, Foschino-Barbaro MP, Cantatore FP. 2010 Nailfold capillaroscopic findings in systemic sclerosis related lung fibrosis and in idiopathic lung fibrosis. Joint Bone Spine

16. Cutolo M, Sulli A, Pizzorni C, Accardo S (2000) Nailfold videocapillaroscopy assessment of microvascular damage in systemic sclerosis. J Rheumatol 27(1):155-160

17. Smith V HAL, Ingegnoli F, Damjanov N, Angelis R D. 2020 standardization of nailfold capillaroscopy for the assessment of the patients with Reynaud's phenomenon and systemic sclerosis. Autoimmunity Reviews

18. Lautenbach G, Dobrota R, Mihai C, Distler O, Calcagni M, Maurer B (2020) Evaluation of botulinum toxin A injections for the treatment of refractory chronic digital ulcers in patients with systemic sclerosis. Clin Exp Rheumatol. 38(Suppl 125(3)):154-60

19. Motegi SI, Uehara A, Yamada K, Sekiguchi A, Fujiwara C, Toki S et al (2017) Efficacy of botulinum toxin B injection for Raynaud's phenomenon and digital ulcers in patients with systemic sclerosis. Acta Derm Venereol 97(7):843-850

20. Motegi S, Yamada K, Toki S, Uchiyama A, Kubota Y, Nakamura T et al (2016) Beneficial effect of botulinum toxin A on Raynaud's phenomenon in Japanese patients with systemic sclerosis: a prospective, case series study. J Dermatol 43(1):56-62

21. Tingey T, Shu J, Smuczek J, Pope J (2013) Meta-analysis of healing and prevention of digital ulcers in systemic sclerosis. Arthritis Care Res (Hoboken) 65(9):1460-1471

22. Wigley FM, Wise RA, Seibold JR, McCloskey DA, Kujala G, Medsger TA Jr. et al (1994) Intravenous iloprost infusion in patients with Raynaud phenomenon secondary to systemic sclerosis. A multicenter, placebo-controlled, double-blind study. Ann Intern Med. 120(3):199-206

23. Neumeister MW (2010) Botulinum toxin type A in the treatment of Raynaud's phenomenon. J Hand Surg Am 35(12):2085-2092

24. Al-Ghamdi AS AN, Joharji H, Al-Qahtani D, Alghamdi H. 2015 Botulinum toxin: non cosmetic and off-label dermatological uses. J Dermatol Dermatolog Surg 
25. Schioppo T, Orenti A, Boracchi P, De Lucia O, Murgo A, Ingegnoli F (2018) Acute and chronic effects of two different intravenous iloprost regimens in systemic sclerosis: a pragmatic nonrandomized trial. Rheumatology (Oxford) 57(8):1408-1416

26. Valentina Cestelli AM, Marco Sebastiani, Emanuela Praino, Francesca Cannarile, Dilia Giuggioli 2016 Clodoveo Ferri Effect of treatment with iloprost with or without bosentan on nailfold videocapillaroscopic alterations in patients with systemic sclerosis. Mod Rheumatol

27. Trombetta AC, Pizzorni C, Ruaro B, Paolino S, Sulli A, Smith $\mathrm{V}$ et al (2016) Effects of longterm treatment with bosentan and iloprost on nailfold absolute capillary number, fingertip blood perfusion, and clinical status in systemic sclerosis. J Rheumatol 43(11):2033-2041
28. Motegi SI, Sekiguchi A, Saito S, Ishibuchi H, Kishi C, Yasuda M et al (2018) Successful treatment of Raynaud's phenomenon and digital ulcers in systemic sclerosis patients with botulinum toxin B injection: assessment of peripheral vascular disorder by angiography and dermoscopic image of nail fold capillary. J Dermatol 45(3):349-352

Publisher's Note Springer Nature remains neutral with regard to jurisdictional claims in published maps and institutional affiliations. 